\title{
Anomalous magnetic moment of the muon with dynam- ical QCD+QED
}

\author{
Alex Westin ${ }^{1, *}$, Waseem Kamleh ${ }^{1,}$, Ross Young ${ }^{1}$, James Zanotti ${ }^{1}$, Roger Horsley ${ }^{2}$, Yoshi- \\ fumi Nakamura ${ }^{3}$, Holger Perlt ${ }^{4}$, Paul Rakow ${ }^{5}$, Gerrit Schierholz ${ }^{6}$, and Hinnerk Stüben ${ }^{7}$, \\ ${ }^{1}$ CSSM, Department of Physics, The University of Adelaide, Adelaide SA 5005, Australia \\ ${ }^{2}$ School of Physics and Astronomy, University of Edinburgh, Edinburgh EH9 3FD, UK \\ ${ }^{3}$ RIKEN Advanced Institute for Computational Science, Kobe, Hyogo 650-0047, Japan \\ ${ }^{4}$ Institut für Theoretische Physik, Universität Leipzig, 04103 Leipzig, Germany \\ ${ }^{5}$ Theoretical Physics Division, Department of Mathematical Sciences, University of Liverpool, Liver- \\ pool L69 3BX, UK \\ ${ }^{6}$ Deutsches Elektronen-Synchrotron DESY, 22603 Hamburg, Germany \\ ${ }^{7}$ Regionales Rechenzentrum, Universität Hamburg, 20146 Hamburg, Germany
}

\begin{abstract}
There exists a long standing discrepancy of around $3.5 \sigma$ between experimental measurements and standard model calculations of the magnetic moment of the muon. Current experiments aim to reduce the experimental uncertainty by a factor of 4 , and Standard Model calculations must also be improved by a similar factor. The largest uncertainty in the Standard Model calculation comes from the QCD contribution, in particular the leading order hadronic vacuum polarisation (HVP). To calculate the HVP contribution, we use lattice gauge theory, which allows us to study QCD at low energies. In order to better understand this quantity, we investigate the effect of QED corrections to the leading order HVP term by including QED in our lattice calculations, and investigate flavour breaking effects. This is done using fully dynamical $\mathrm{QCD}+\mathrm{QED}$ gauge configurations generated by the QCDSF collaboration and a novel method of quark tuning.
\end{abstract}

\section{Introduction}

The anomalous magnetic moment of the muon, $a_{\mu}=\frac{g-2}{2}$, is one of the most precisely measured quantities in particle physics [1]. Despite the precision, there currently exists a $3.5-4$ standard deviation discrepancy between theoretical Standard Model (SM) predictions and experimental determinations. This persistent discrepancy leads the anomalous magnetic moment of the muon to be considered as a potential candidate to observe physics beyond the Standard Model [2]. At present, the experimental Ref. [3] uncertainty and the total theoretical uncertainties are of comparable magnitude. The Muon $g-2$ Experiment at Fermilab Ref. [4] aims to reduce the experimental uncertainty to 140 parts-per-billion, a 4 times improvement. Thus it is essential to get the theoretical uncertainties down to a comparable precision. It is well known that the theoretical uncertainty of $a_{\mu}$ is dominated by the hadronic contribution, in particular the Hadronic Vacuum Polarisation (HVP) and hadronic light-by-light scattering

\footnotetext{
*Speaker. e-mail: alex.westin@adelaide.edu.au
} 


\begin{tabular}{l|llllllll} 
Ensemble & $L^{3} \times T$ & $N_{f}$ & $m_{u \bar{u}}$ & $m_{d \bar{d}}$ & $m_{s \bar{s}}$ & $m_{q \bar{q}}^{\min } L$ & $m_{\pi^{+}}$ & $m_{K^{+}}$ \\
\hline \hline 1 & $32^{3} \times 64$ & $2+1$ & 430 & 405 & 405 & 4.4 & 435 & 435 \\
2 & $32^{3} \times 64$ & $2+1$ & 360 & 435 & 435 & 4.0 & 415 & 415 \\
3 & $32^{3} \times 64$ & $1+1+1$ & 290 & 300 & 585 & 3.2 & 320 & 470 \\
4 & $48^{3} \times 96$ & $2+1$ & 430 & 405 & 405 & 6.7 & 435 & 435 \\
5 & $48^{3} \times 96$ & $2+1$ & 360 & 435 & 435 & 5.9 & 420 & 420 \\
6 & $48^{3} \times 96$ & $1+1+1$ & 290 & 300 & 580 & 4.8 & 320 & 470
\end{tabular}

Table 1: Ensembles used in this work. All masses are in MeV.

contributions ([5]). In this study we will focus on the HVP term. Currently, the best results for HVP come from $e^{+} e^{-}$scattering cross sections, which contribute a $0.5 \%$ uncertainty. Lattice simulations of the HVP term have received a surge of interest over the past few years, with results now being quoted at the physical point with $\mathscr{O}(1 \%)$ errors $[6,7]$, and are predicted to soon offer the most precise results for the HVP term. Traditionally, these lattice calculations have only been done using pure QCD, or have included QED though various methods such as partially quenched QED. As we are pushing for a very high level of precision, the question arises of whether we need to start including the full electromagnetic (EM) corrections to our lattice calculations of the HVP term. In this work we employ six ensembles of fully dynamical QCD+QED lattice configurations generated by the QCDSF collaboration, including simulations on two different volumes, shown in Table 1 . These ensembles are tuned using a novel scheme such that the average quark mass is kept constant at the average physical quark mass using the Dashen scheme, as described in Ref. [8].

\section{Time-moment representation}

We use the time-momentum representation proposed in Ref. [9] to calculate the HVP contribution to the anomalous magnetic moment of the muon. We can calculate $a_{\mu}^{H V P}$ from the vacuum subtracted polarisation function $\hat{\Pi}\left(Q^{2}\right)$ using

$$
a_{\mu}^{H V P}=\left(\frac{\alpha}{\pi}\right)^{2} \int_{0}^{\infty} d Q^{2} K\left(Q^{2} ; m_{\mu}^{2}\right) \hat{\Pi}\left(Q^{2}\right)
$$

where $K\left(Q^{2} ; m_{\mu}^{2}\right)$ is a known kernel function (Ref. [10]),

$$
\begin{aligned}
K\left(Q^{2} ; m_{\mu}^{2}\right) & =m_{\mu}^{2} Q^{2} Z^{3}\left(Q^{2}\right) \frac{1-Q^{2} Z\left(Q^{2}\right)}{1+m_{\mu}^{2} Q^{2} Z^{2}\left(Q^{2}\right)}, \\
Z\left(Q^{2}\right) & =\left(\sqrt{\left(Q^{2}\right)^{2}+4 m_{\mu}^{2} Q^{2}}-Q^{2}\right) /\left(2 m_{\mu}^{2} Q^{2}\right) .
\end{aligned}
$$

In the time-moment representation, the vacuum subtracted polarisation function, $\hat{\Pi}\left(Q^{2}\right)$, is obtained from the spatially summed two-point correlator, $G(t)$,

$$
\begin{aligned}
\hat{\Pi}\left(Q^{2}\right) & =4 \pi^{2} \int_{0}^{\infty} d t G(t)\left(t^{2}-\frac{4}{Q^{2}} \sin ^{2}\left(\frac{Q t}{2}\right)\right), \\
G(t) & =-\int d^{3} x\left\langle J_{i}(x) J_{i}(0)\right\rangle
\end{aligned}
$$


where

$$
J_{i}(x)=\frac{2}{3} \bar{u}(x) \gamma_{i} u(x)-\frac{1}{3} \bar{d}(x) \gamma_{i} d(x)-\frac{1}{3} \bar{s}(x) \gamma_{i} s(x)+\ldots
$$

Substituting this into Equation 1, we can rewrite it as

$$
a_{\mu}^{H V P}=\left(\frac{\alpha}{\pi}\right)^{2} \int_{0}^{\infty} d t G(t) \tilde{K}\left(t ; m_{\mu}\right)
$$

where $\tilde{K}\left(t ; m_{\mu}\right)$ is given by

$$
\tilde{K}\left(t ; m_{\mu}\right)=4 \pi^{2} \int_{0}^{\infty} d Q^{2} K\left(Q^{2} ; m_{\mu}\right)\left[t^{2}-\frac{4}{Q^{2}} \sin ^{2}\left(\frac{Q t}{2}\right)\right],
$$

and $K\left(Q^{2} ; m_{\mu}\right)$ is the same kernel function as in Equation 1. Here we employ the analytic form for $\tilde{K}\left(t ; m_{\mu}\right)$ derived in Ref. [11].

\section{Results and discussion}

\subsection{Time Moment}

We calculate $a_{\mu}^{H V P}$ from Equation 7, following the method outlined in Ref. [11]. At large times, the 2 point function $G(t)$ suffers from a loss of signal into statistical noise, and is contaminated by the backwards propagating state. Since Equation 7 requires $G(t)$ to be known to infinite time, this issue is overcome by only using the 2 point function data, $G^{\text {data }}(t)$ up to some value of $t=t_{\text {cut }}$. At this point we perform a cut, after which we replace the lattice correlator with a fitted form in order to extrapolate to large time. For this fit we use a series of exponentials to represent the correlator, such that

$$
G(t)= \begin{cases}G^{\text {data }}(t) & t \leq t_{c u t}, \\ \sum_{i} A_{i} e^{-E_{i} t} & t>t_{\text {cut }}\end{cases}
$$

The first exponential in the series represents the ground state, and the second and subsequent exponentials represent the first excited state and so on. We have performed these fits with 1,2 and 3 states, that is, the ground state and up to 2 excited states included. These fits are performed on a window starting at $t_{c u t}$, and extending until the signal is lost to noise or the backwards propagating state, based on a covariance $\chi^{2}$ metric.

As multiple exponential fits can be unstable on some of the ensembles considered, we choose to use the 1 stage fit for our analysis. This also takes advantage of the lower statistical uncertainty of our lattice data at early Euclidean times $t$. We use the 2 and 3 state fits to ensure that our 1 state fit window has minimal excited state contamination. Figure 1a shows that the ground state dominates the signal as long as $t_{c u t}$ is sufficiently large. By choosing values of $t_{\text {cut }}$ large enough we can accurately represent the data using a 1 state fit with little excited state contamination.

For the region $t<t_{c u t}$ we use a cubic spline over the lattice data to compute the contribution of this region to the integral in Equation 7.

An example of our fitting method is shown in Figure 1b, where we have used the raw lattice data before $t_{c u t}$, and then our exponential fit after. The datapoints in grey show the lattice data after $t_{\text {cut }}$, which are not used. These are closely reflected in the fit within the fit window, but are dominated by the backwards propagating state towards the larger time values. Note that this is a plot of the full integrand in Equation 7, not just the correlator $G(t)$.

Repeating this fitting scheme over all our ensembles, we can then calculate $a_{\mu}^{H V P}$ for all our different quark charges and masses. We use the meson mass flavour-breaking expansion 


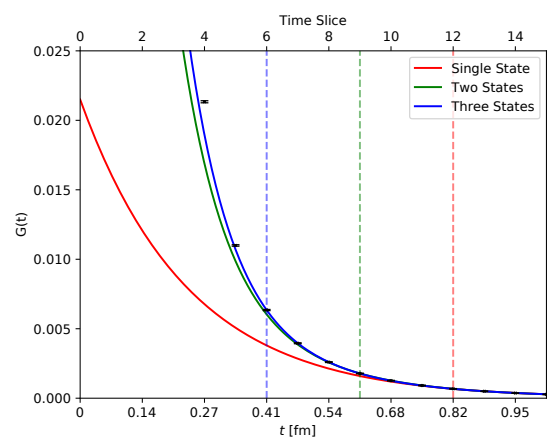

(a) 1, 2 and 3 state fits, with the earliest timeslice included in the fit $\left(t_{c u t}\right)$ for each fit shown by the dashed line. Correlator data is shown by black markers. Note that higher state contribution is minimal after the cut point.

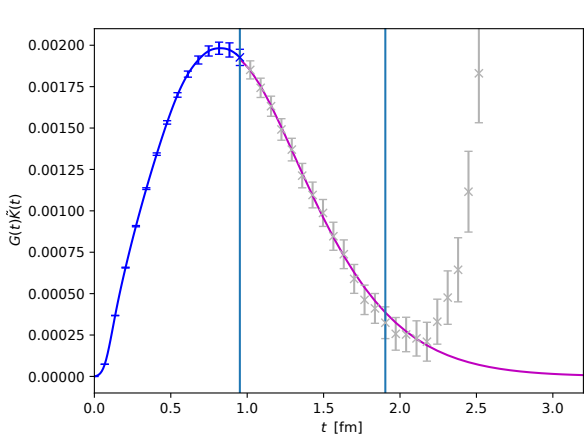

(b) Plot showing the full integrand of Equation 7. Blue crosses are the integrand data points used in constraining a cubic spline (blue curve). The magenta line in the tail is from our exponential function fit for the $t>t_{c u t}$ region, with the fit window indicated by the two vertical lines Greyed crosses are integrand points after $t_{c u t}$, which are replaced by the fitted exponential.

Figure 1

detailed in Refs. [8] and [12], adapted for the SU(3)-flavour properties of $a_{\mu}^{H V P}$ to extrapolate to the physical masses.

Figure 2 shows our values for $a_{\mu}^{H V P}$ plotted against the Dashen mass, $\mu_{q}^{D}$. Note that for ease of plotting, we have compressed the direction relevant to the variation of $a_{\mu}^{H V P}$ with sea quark mass by shifting all points to the physical sea quark masses $\delta m_{q}=\delta m_{q}^{p h y s}$. The physical values for the valence quark masses are given by the red (up), green (down) and blue (strange) vertical dashed lines. The final value for $a_{\mu}^{H V P}$ is obtained by taking the appropriate charge-weighted combination of all three quark flavour contributions at their physical masses,

$$
a_{\mu}^{H V P}=\frac{4}{9}(u)+\frac{1}{9}(d)+\frac{1}{9}(s) .
$$

By doing this we find a value for $a_{\mu}$ on each volume. For the $32^{3} \times 64$ volume we find $a_{\mu}^{H V P, L O}=492 \pm 15 \times 10^{-10}$, and on the $48^{3} \times 96$ volume $a_{\mu}^{H V P, L O}=579 \pm 23 \times 10^{-10}$. The difference between the two volumes is not unexpected due to the significant finite volume effects mentioned in Ref. [9, 13].

Even so, we still find the value on our larger volume to be smaller than expected when comparing to other lattice studies using similar setups and methods. However, as finite volume corrections are not included, we estimate the consistency of our results with other studies by comparing to those other results on a similar volume, without finite volume corrections applied. Ref [11] find $\left(a_{\mu}^{H V P, L O}\right)^{u d s}=602.4(24.8)(28.9) \times 10^{-10}$ before applying finite volume corrections which is consistent with our results. 

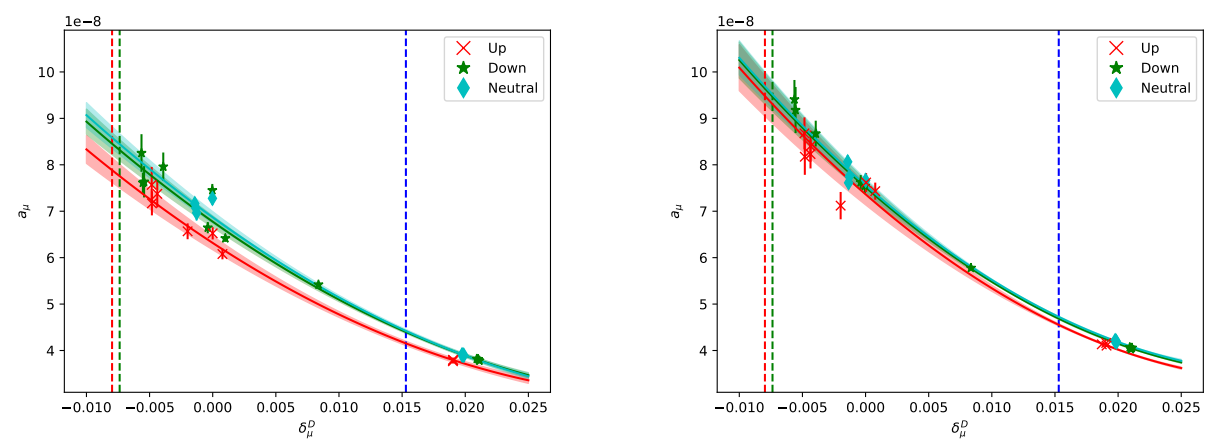

Figure 2: $a_{\mu}^{H V P}$ against Dashen mass, left: $32^{3} \times 64$, right: $48^{3} \times 96$. Note that points are shifted to $\delta m=\delta m^{\text {phys }}$ line. Colours refer to quarks with different charges, red: Up quark, green: Down/strange quarks, cyan: 'Neutral' quark. Vertical lines mark the physical quark mass

\subsection{Charge Contribution}

We can note in Figure 2 the difference in slopes between the red (up quarks with charge $+\frac{2}{3} e$ ) and green (down/strange quarks with charge $-\frac{1}{3} e$ ) curves, which is purely an EM effect. To quantify the significance of this effect we can look at the deviation of the charged curves from the neutral (in blue, using quarks with charge $0 e$ ) curve, and normalising it against the neutral curve. We do this in Figure 3 by taking the ration $\frac{a_{\mu}^{u}-a_{\mu}^{n}}{a_{\mu}^{n}}$, and similar for the down quark. A quantitative result will be coming in a future publication soon, but it is clear that there is a non-zero EM effect due to quark charge.

As the aim is for lattice to reach a level of precision with errors less than $1 \%$, controlling these EM effects looks to be of increasing importance going forwards.
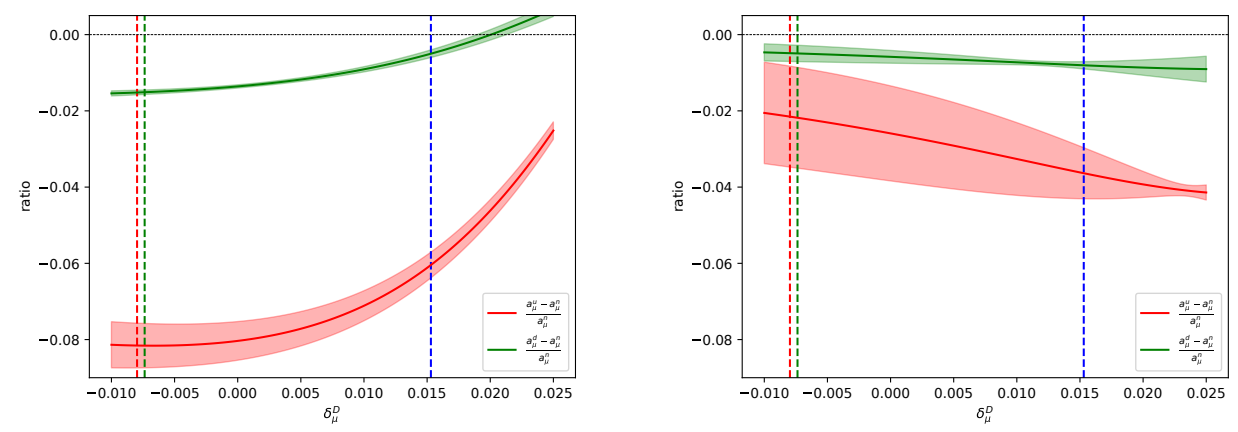

Figure 3: Deviation of charged contribution from neutral, normalised to the neutral contribution, on each volume. left: $32^{3} \times 64$, right: $48^{3} \times 96$ 


\section{Acknowledgements}

The numerical configuration generation (using the BQCD lattice QCD program Ref. [14]) and data analysis (partly using the Chroma software library Ref. [15]) was carried out on the IBM BlueGene/Q and HP Tesseract using DIRAC 2 resources (EPCC, Edinburgh, UK), the IBM BlueGene/Q at NIC (Jülich, Germany), the Cray XC40 at HLRN (The North-German Supercomputer Alliance), the NCI National Facility in Canberra, Australia, and the iVEC facilities at the Pawsey Supercomputing Centre. These Australian resources are provided through the National Computational Merit Allocation Scheme and the University of Adelaide Partner Share supported by the Australian Government. This work was supported in part through supercomputing resources provided by the Phoenix HPC service at the University of Adelaide. AW was supported by an Australian Government Research Training Program Scholarship. HP was supported by DFG Grant No. PE 2792/2-1, PELR in part by the STFC under contract ST/G00062X/1 and RDY and JMZ by the Australian Research Council under grants FT120100821, FT100100005, and DP140103067.

\section{References}

[1] F. Jegerlehner, A. Nyffeler, Physics Reports 477, 1 (2009), 0902.3360

[2] M. Tanabashi et al. (Particle Data Group), Phys. Rev. D 98, 030001 (2018)

[3] G.W. Bennett et al. (Muon g-2), Phys. Rev. D73, 072003 (2006), hep-ex/ 0602035

[4] A. Chapelain (Muon g-2), EPJ Web Conf. 137, 08001 (2017), 1701.02807

[5] M.K. Marinkovic, Few Body Syst. 58, 140 (2017), 1704.06425

[6] K. Miura, PoS LATTICE2018, 010 (2019), 1901.09052

[7] S. Borsanyi et al. (2020), 2002.12347

[8] R. Horsley et al., JHEP 04, 093 (2016), 1509.00799

[9] D. Bernecker, H.B. Meyer, Eur. Phys. J. A47, 148 (2011), 1107.4388

[10] T. Blum, Phys. Rev. Lett. 91, 052001 (2003), hep-lat/ 0212018

[11] M. Della Morte et al., JHEP 10, 020 (2017), 1705.01775

[12] R. Horsley et al., J. Phys. G43, 10LT02 (2016), 1508 . 06401

[13] C. Aubin et al., Phys. Rev. D93, 054508 (2016), 1512.07555

[14] T.R. Haar, Y. Nakamura, H. Stüben, EPJ Web Conf. 175, 14011 (2018), 1711.03836

[15] R.G. Edwards, B. Joo, Nucl. Phys. Proc. Suppl. 140, 832 (2005), hep-lat / 0409003 